\title{
Is confounding considered when estimating treatment-covariate interactions in individual participant data meta-analyses of randomised trials? A methodological study
}

\author{
Ewelina Rogozinska ${ }^{1}$, Chantelle Cornett ${ }^{1}$, Nadine Marlin², David Fisher ${ }^{1}$, Peter J Godolphin ${ }^{1}$
}

Corresponding Author: Peter J Godolphin, $2^{\text {nd }}$ Floor, 90 High Holborn, MRC Clinical Trials Unit at University College London, Institute of Clinical Trials and Methodology, London, WC1V 6LJ, UK. p.godolphin@ucl.ac.uk

\author{
Affiliations \\ 1: MRC Clinical Trials Unit at University College London, Institute of Clinical Trials and Methodology, London, \\ UK \\ 2: Pragmatic Clinical Trials Unit, Barts and The London School of Medicine and Dentistry, Queen Mary \\ University of London, London, UK
}

\section{Background}

A key question for meta-analyses is reliably assessing whether treatment effects vary across different participant groups, thereby informing how best to treat individual patients. A substantial benefit of the increase in participant numbers afforded by meta-analysis is that there is sufficient power to estimate interactions between treatments and participant covariates (e.g., disease burden in prostate cancer) ${ }^{1}$.

However, it is possible that evidence of an interaction is driven by a different, confounding factor. This may be a particular problem for cancer meta-analyses, where patients have several similar baseline variables collected (e.g., Cancer stage, Nodal status, Volume of disease etc.). In an individual patient data (IPD) metaanalysis, it might be possible to account or adjust for any potential confounding. A research question of interest is: what is the most appropriate way of dealing with potentially confounding factors when estimating treatment-covariate interactions in an IPD meta-analysis of randomised trials? However, first, we need to understand what methods exist to deal with confounding and what methods are currently being used in practice. 


\section{Objectives}

The objectives of our work are to:

1. Evaluate published reports of IPD meta-analyses of randomised trials for the type of approach adopted to assess treatment-covariate interactions, specifically whether confounding factors were accounted for when estimating interactions.

2. Evaluate the reasons why specific analyses were chosen for any interaction analyses that are different from a model containing only the treatment-covariate interaction.

3. Identify if methods and approaches differ for one-stage and two-stage models.

4. In the studies where the confounding was accounted for, identify the theoretical concepts underpinning applied methodology.

\section{Methods}

\section{Literature search}

This project is related to a similar project exploring non-linear associations when estimating treatmentcovariate interactions in IPD meta-analyses ${ }^{2}$. Both projects share the same search strategy, and this strategy identifies IPD meta-analyses published between 2015 and 2020. Eligible articles for our project will be IPD meta-analyses of at least two randomised trials in which at least one treatment-covariate interaction is reported. Articles will be screened for eligibility by one reviewer, with another reviewer independently assessing $10 \%$ of records for eligibility. Additionally, we will look for theoretical papers underpinning the applied methodology (these are additional to the "eligible articles" mentioned previously).

\section{Sample size}

We will take a random sample of 100 eligible articles for this project. Our approach to selecting a random sample will be to screen the $10^{\text {th }}$ record until we have identified 100 eligible articles. The justification for this number is that it enables a timely and thorough assessment of the current literature, without being too resource intensive. Note that we do not attempt to describe the approaches of all IPDs between 2015 and 
2020, but instead aim to give a representative picture of the current practice in IPD meta-analyses. We will supplement this number with additional theoretical papers as appropriate to meet objective 4. Experience with a similar project using the same search strategy ${ }^{2}$ that assessed a random sample of 100 found that data saturation occurred around article 80.

\section{Data collection}

We will use a bespoke data collection form that will be tested on five eligible studies. The same form is being used in the aforementioned similar project ${ }^{1}$. We will collect the following information for our study:

- Contact details for the corresponding author, principal investigator, and the lead statistician

- Paper's characteristics:

- publication year,

- medical area,

- number of trials with available IPD and a corresponding total number of participants.

- Details of the evaluated interactions:

- number of patient-level interactions reported,

- number of outcomes considered for effect modification,

- type of covariate (continuous, categorised continuous, categorical),

- type of outcome:

$\circ$ binary, time-to-event, continuous

- composite (yes/no)

- Details on implemented statistical models

- one/two stage,

- fixed-effect/random-effects,

- general modelling approach,

- analysis approach for investigation of effect modification,

- Inclusion of any additional covariates beyond the treatment-effect modification,

- Selection and approach to inclusion of additional covariates,

- Reasons for inclusion of additional covariates. 
Data will be extracted by a single reviewer and $10 \%$ of the extracted studies cross-checked independently by a further reviewer. Disagreements between reviewers will be resolved through discussion with the wider research team as appropriate. If multiple treatment-covariate interactions are reported, then we will focus on any that include additional covariates beyond the treatment-covariate interaction, although we will record the number of other reported interactions. Extracted data will be collated in an Excel spreadsheet.

Additional documents will be sought (protocol, statistical analysis plan etc.) to supplement the data extraction as appropriate. If the authors' stated in their publication that the interaction was adjusted but will not provide details on how the adjustment was made, and the statistical analysis plan is not available in the public domain (as an appendix or online document), we will contact one of the team members (ideally the statistician or other relevant person (e.g., $\mathrm{Pl} /$ methodologist) to obtain this information).

\section{Analysis}

All analysis will be descriptive using Stata software (version 16.1). In addition to describing current practice, we will describe the various methodological approaches to account for confounding when estimating patientlevel treatment-covariate interactions that we identify from the eligible articles, and that are further supplemented through additional theoretical papers.

\section{References}

[1] Fisher D J, Carpenter J R, Morris T P, Freeman S C, Tierney J F. Meta-analytical methods to identify who benefits most from treatments: daft, deluded, or deft approach? BMJ 2017; 356 :j573 doi:10.1136/bmj.j573

[2] Marlin N. The analysis of a complex association in an individual participant data meta-analysis: protocol for a methodological review. PROSPERO 2019 CRD42019126768 Available from:

https://www.crd.york.ac.uk/prospero/display record.php?ID=CRD42019126768

\section{Competing interests}

No competing interests exist 


\section{Funding}

ER and DF are supported by the UK Medical Research Council (https://mrc.ukri.org/) Grant number: MC_UU_00004/06. CC is supported through a National Institute for Health Research (NIHR) Methodology Internship. NM is supported by a NIHR Doctoral Research Fellowship (DRF-2018-11-ST2-077). PJG and DJF are part supported by Prostate Cancer UK (https://prostatecanceruk.org/) Grant number: RIA 16-ST2-020. PJG is part supported by a NIHR Development and Skills Enhancement Award (NIHR301653). The views expressed in this publication are those of the author(s) and not necessarily those of the UK Medical Research Council, the NHS, the NIHR or the UK Department of Health. 